ARTIGO ORIGINAL

ORIGINAL ARTICLE

\section{Custo-efetividade de mirabegrona no tratamento de primeira escolha da síndrome da bexiga hiperativa: uma análise sob a perspectiva do sistema de saúde brasileiro}

\author{
Cost-effectiveness of mirabegron as first-choice \\ treatment for overactive bladder syndrome: an analysis \\ from the Brazilian health system perspective \\ Cristiano Gomes ${ }^{1}$, Ricardo Vita², Caio Cintra 3 , Sarah Gonçalves ${ }^{4}$, Roberto Soler ${ }^{4}$
}

DOI: $10.21115 / J B E S . v 12 . n 1 . p 56-65$

\begin{abstract}
Palavras-chave:
bexiga urinária, bexiga hiperativa, agonistas beta-3-adrenérgicos, antimuscarínicos, análise custo-efetividade
\end{abstract}

\section{RESUMO}

Objetivo: Avaliar a relação de custo-efetividade de mirabegrona como tratamento de primeira escolha de pacientes adultos com síndrome da bexiga hiperativa (SBH) comparada a antimuscarínicos orais comumente prescritos no manejo dessa condição. Métodos: $\bigcirc$ modelo de Markov foi utilizado, com ciclos mensais e horizonte temporal de um ano, para analisar a relação de custo-efetividade de mirabegrona em comparação a tolterodina, oxibutinina, darifenacina e solifenacina. Os pacientes iniciaram o modelo em tratamento com mirabegrona ou um dos comparadores, sendo distribuídos em cinco níveis de gravidade da doença, de acordo com a frequência miccional e número de episódios de incontinência, e a cada ciclo mensal poderiam melhorar, piorar ou permanecer no mesmo nível de severidade do ciclo anterior. Os resultados foram apresentados por meio de uma razão de custo-efetividade incremental. Resultados: Considerando a perspectiva do sistema de saúde suplementar, o custo total do tratamento com mirabegrona foi de R\$ 2.455,26 e os parâmetros de efetividade em 0,491 e 0,498 (melhora na gravidade da incontinência e frequência miccional, respectivamente), sendo mais efetivo que os comparadores. Com relação à perspectiva pública, foi estimado um custo com o tratamento de $\mathrm{R} \$ 1.396,01$, com características similares de efetividade. De maneira geral, o tratamento com mirabegrona foi dominante quando comparado à tolterodina e custo-efetivo na comparação com os demais antimuscarínicos, em ambas as perspectivas analisadas. Conclusão: Mirabegrona demonstrou ser a melhor opção para tratamento de primeira escolha da SBH com potencial de redução de custos ao longo do tempo, tanto para o sistema público quanto para o sistema de saúde suplementar brasileiro.

\section{ABSTRACT}

Objective: To assess the cost-effectiveness of mirabegron as first-choice treatment in adult patients with of overactive bladder (OAB) compared to oral antimuscarinics, usually prescribed for this condition. Methods: A Markov model has been adopted, with monthly cycles and a one-year time horizon, to analyze the cost-effectiveness of mirabegron compared to antimuscarinic agents: tolterodine; oxybutynin; darifenacin; and solifenacin. The model started with patients receiving treatment with mirabegron or one of the comparators and then, they were assigned to five disease severity levels according to micturition frequency and number of incontinence episodes, and within each monthly cycle they could improve, worsen or remain at the same symptom severity level. Results were presented using an incremental cost-effectiveness ratio. Results: Considering the Brazilian pri-

\footnotetext{
Recebido em: 03/03/2020. Aprovado para publicação em: 15/03/2020.

1. Divisão de Urologia, Hospital das Clínicas da Faculdade de Medicina da Universidade de São Paulo, São Paulo, SP, Brasil.

2. Clínica de Urologia, Hospital Militar de Área de São Paulo do Exército Brasileiro, São Paulo, SP, Brasil.

3. Disciplina de Urologia da Faculdade de Medicina do ABC, Santo André, São Paulo, SP, Brasil.

4. Astellas Farma Brasil, São Paulo, SP, Brasil.

Instituição onde o trabalho foi executado: SENSE Company, São Paulo, SP, Brasil.

Informações sobre auxílios recebidos sob a forma de financiamento, equipamentos ou medicamentos: Este estudo

foi financiado pela Astellas Farma Brazil.

Congressos onde o estudo foi apresentado: Não foi apresentado até o momento.

Conflitos de interesse: Gonçalves S. e Soler R. são funcionários da Astellas.

Autor correspondente: Roberto Soler. Av. Dr. Chucri Zaidan, 1.170, Brooklin Novo, São Paulo, SP. CEP: 04583-110. Telefone: +55 (11)
} 3027-4543. E-mail roberto.soler@astellas.com 
vate perspective, treatment with mirabegron resulted in a total cost of $R \$ 2,455.26$ and effectiveness parameters at 0.491 and 0.498 (improvement in incontinence severity and micturition, respectively), being more effective than the comparators. Regarding the Brazilian public perspective, treatment with mirabegron resulted in a total cost of $\mathrm{R} \$ 1,396.01$ with similar effectiveness estimation. In general, a dominance was observed when mirabegron was compared to tolterodine and a cost-effectiveness profile against the other muscarinic antagonists, considering both health perspectives. Con-

clusion: Mirabegron has proven to be the best option for $O A B$ first-line treatment with potential cost savings over time for both the public and private health care systems in Brazil.

\section{Introdução}

A síndrome da bexiga hiperativa (SBH) é caracterizada pela presença de urgência urinária com ou sem incontinência de urgência, geralmente acompanhada de aumento da frequência miccional diurna e noctúria [International Continence Society (ICS), 2018]. Estima-se que, em 2018, 546 milhões de indivíduos apresentavam essa síndrome em todo o mundo (Irwin et al., 2011). No Brasil, um estudo populacional conduzido em cinco grandes capitais demonstrou que 25\% dos homens e $24 \%$ das mulheres apresentam sintomas de SBH (Soler et al., 2018).

A SBH afeta negativamente a qualidade de vida relacionada à saúde (QVRS), além de impactar negativamente aspectos sociais, psicológicos, ocupacionais, domésticos, físicos e sexuais dos pacientes que vivenciam a doença (Abrams et al., 2000; Gomes et al., 2019; Johnston et al., 2019; Soler et al., 2019; Tubaro, 2004; Gomes et al., 2020). Além disso, a SBH pode impactar economicamente indivíduos e sociedade. O custo da síndrome representa uma carga econômica e social substancial, com custos médicos diretos específicos para o seu manejo variando entre 656 e 860 dólares americanos (USD) por paciente por ano nos Estados Unidos e custos indiretos totais de 11.134 USD por paciente por ano no mesmo país (Irwin et al., 2009; Powell et al., 2018).

O tratamento de primeira linha da SBH inclui a terapia comportamental, que visa à educação e à orientação dos pacientes para o desenvolvimento de estratégias de gerenciamento da urgência e incontinência. Tais estratégias envolvem, entre outros, alterações na dieta e ingestão de líquidos, regularização do hábito intestinal, micções pelo relógio ou programadas, perda de peso e exercícios para o assoalho pélvico, com ou sem a utilização de biofeedback e eletroestimulação (Leron et al., 2018).

O tratamento farmacológico, considerado como estratégia de segunda linha, é baseado no uso de antimuscarínicos e agonistas beta-3-adrenérgicos. O objetivo principal do tratamento é promover o relaxamento do músculo detrusor, aliviando os sintomas (Corcos et al., 2017; Leron et al., 2018; Lightner et al., 2019). Apesar da eficácia comprovada e da disponibilidade de antagonistas com maior seletividade para receptores muscarínicos da bexiga, eventos adversos (EAs) são frequentes com o uso dessa classe de medicamentos. Tais eventos ocorrem principalmente pelo bloqueio de receptores muscarínicos em ou- tros órgãos do corpo. Os principais EAs relatados incluem boca seca, constipação e visão turva. EAs relacionados ao sistema nervoso central (SNC) também podem ocorrer, tais como alterações de memória, tontura e sonolência, notadamente em indivíduos mais idosos e fragilizados. A ocorrência de EAs é uma das principais causas de baixa adesão e/ou abandono do tratamento na SBH, assim como em outras doenças crônicas (Leron et al., 2018; Ricetto Sacomani et al., 2019).

A mirabegrona é o primeiro agonista de receptores beta-3-adrenérgicos aprovado para o tratamento da SBH e o único disponível comercialmente. Por meio da estimulação dos receptores beta-3-adrenérgicos, a mirabegrona promove o relaxamento da bexiga, melhorando o armazenamento da urina e reduzindo contrações vesicais e, consequentemente, os sintomas da SBH (Deeks, 2018). Além disso, devido ao seu mecanismo de ação, os agonistas beta-3-adrenérgicos não estão associados aos EAs típicos dos antimuscarínicos. Os EAs mais comuns com o uso de agonistas beta-3-adrenérgicos são hipertensão e cefaleia, com incidências semelhantes às reportadas com placebo (Nitti et al., 2013; Deeks, 2018). Em estudos clínicos, a mirabegrona mostrou-se eficaz em 12 semanas e em 12 meses, sendo capaz de reduzir a frequência miccional e a incontinência urinária, além de demonstrar benefícios clínicos como redução da urgência para urinar, aumento do volume miccional e melhora da QVRS. Adicionalmente, esse fármaco mostrou bom perfil de tolerabilidade e segurança, com dados de diferentes países que indicam melhor persistência e adesão ao tratamento, quando comparado aos antimuscarínicos (Nitti et al., 2013; Kelleher et al., 2018; Deeks, 2018).

Pacientes que não obtêm melhora clínica com a terapia comportamental e com o tratamento farmacológico, ou que não são candidatos para essas terapias, podem ser considerados para tratamentos invasivos de terceira linha. Entre essas estratégias, encontram-se a injeção de toxina botulínica no músculo detrusor, a eletroestimulação percutânea do nervo tibial posterior e a neuromodulação sacral. Raramente, pode ser necessária a realização de cirurgia para ampliação vesical, usando segmento intestinal (Corcos et al., 2017; Lightner et al., 2019).

Dada a disponibilidade de diferentes estratégias farmacológicas para o tratamento da doença, análises que ponderam a relação entre a eficácia e os custos de cada estratégia são essenciais para o processo de tomada de decisão (Secoli et al., 
2010). Dessa forma, este estudo foi conduzido com o objetivo de avaliar a relação de custo-efetividade das diferentes opções de tratamento farmacológico para SBH no Brasil.

\section{MÉTODOS}

\section{Visão geral da análise}

Um modelo de Markov foi desenvolvido em Microsoft Excel, versão 2016, para analisar a relação de custo-efetividade de mirabegrona 50 mg, uma vez ao dia, em comparação aos agentes antimuscarínicos registrados no país para o manejo da SBH em primeira escolha de tratamento:

- Tolterodina de liberação estendida: 4 mg uma vez ao dia;

- Oxibutinina de liberação estendida: 10 mg uma vez ao dia;

- Oxibutinina de liberação imediata: $5 \mathrm{mg}$ duas vezes ao dia;

- Darifenacina: 7,5 mg ou 15 mg uma vez ao dia;

- Solifenacina: 5 mg ou 10 mg uma vez ao dia.

O modelo simulou o manejo terapêutico e o curso natural da doença, bem como as complicações relativas ao tratamento e seu impacto em custos e desfechos. O desfecho da análise foi medido pela melhora do nível de gravidade da doença em termos de frequência de micção e episódios de incontinência. O modelo adotou ciclos mensais e um horizonte de tempo de um ano. Esse horizonte é justificado pelo fato de que dados de mundo real sugerem que, em média, $75 \%$ a $90 \%$ dos pacientes descontinuam a terapia de primeira linha em até um ano (Wagg et al., 2017). O modelo assumiu que não há troca de tratamento. Dessa forma, pacientes que descontinuam a medicação permanecem sem tratamento, não iniciando uma estratégia terapêutica subsequente. Essa escolha foi realizada para evitar maior complexidade da análise, além de evitar o impacto causado por diferentes sequências de tratamento em custos e desfechos. Dessa forma, assegura-se que os resultados da análise são decorrentes exclusivamente do tratamento farmacológico de primeira escolha analisado. Foram adotadas tanto a perspectiva do sistema de saúde público quanto a do sistema de saúde suplementar brasileiro.

\section{Estrutura do modelo}

Os pacientes iniciaram o modelo em tratamento com mirabegrona ou um dos comparadores selecionados, sendo distribuídos em cinco níveis de gravidade da doença, de acordo com a frequência miccional e o número de episódios de incontinência. A cada ciclo mensal poderiam melhorar (transitar para um estado de menor gravidade da doença), piorar (estado de maior gravidade) ou permanecer no mesmo nível do ciclo anterior (Figura 1). De acordo com o nível de gravidade da doença, poderiam haver a ocorrência de comorbidades e a

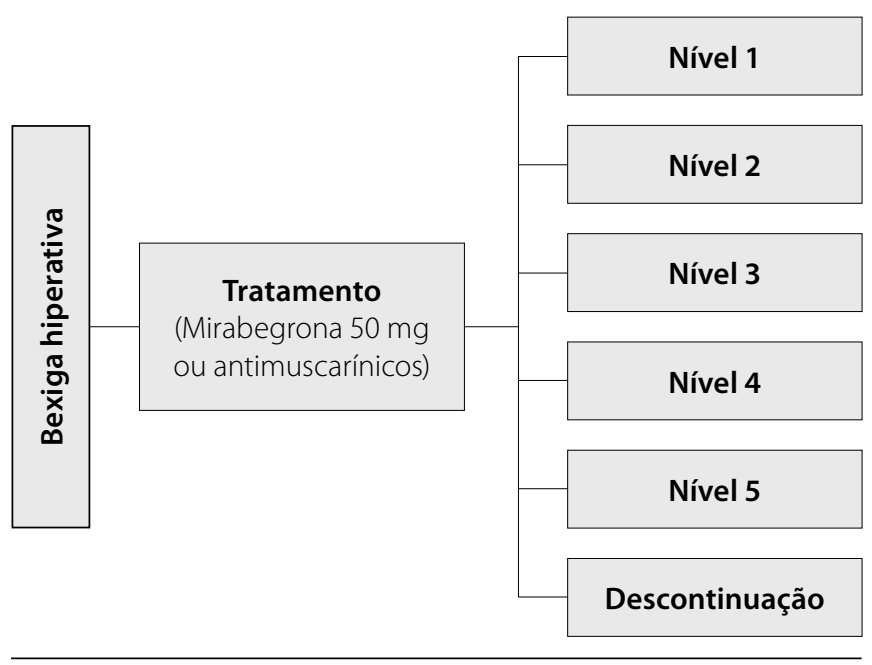

Figura 1. Estrutura simplificada do modelo.

necessidade do uso de absorventes para incontinência. Além disso, os pacientes permaneciam sob risco de EAs e das consequências da carga anticolinérgica relativa ao tratamento com antimuscarínicos. O modelo considerou taxas de descontinuação específicas para cada medicamento de acordo com dados encontrados na literatura (Chapple et al., 2017).

Os custos incluíram a aquisição de medicamentos, consultas, monitoramento, comorbidades e EAs relacionados à doença e à carga anticolinérgica, bem como a aquisição de absorventes para incontinência. Os resultados foram apresentados por meio da razão de custo-efetividade incremental (RCEI), que corresponde ao custo incremental em relação ao benefício promovido pela intervenção (em relação à melhora do nível de gravidade da doença medido pela frequência miccional e número de episódios de incontinência).

\section{Parâmetros do modelo}

\section{Dados basais}

As distribuições de idade e gênero da coorte hipotética foram extraídas do estudo Brazil LUTS, apresentadas na Tabela 1 (Soler et al., 2018). Os níveis de gravidade inicial dos sintomas basearam-se em dados do estudo SCORPIO, apresentados na Tabela 2 (Khullar et al., 2013).

Tabela 1. Distribuição inicial dos pacientes por sexo e idade (Soler et al., 2018)

\begin{tabular}{lcc}
\hline Faixa etária & Homens, \% & Mulheres, \% \\
\hline $20-44$ & 7,3 & 6,9 \\
\hline $45-54$ & 15,3 & 16,0 \\
\hline $55-64$ & 12,8 & 14,7 \\
\hline $65-74$ & 8,2 & 10,5 \\
\hline $75+$ & 3,4 & 4,9 \\
\hline
\end{tabular}


Tabela 2. Distribuição inicial dos pacientes por nível de gravidade da doença (Khullar et al., 2013)

\begin{tabular}{|c|c|c|c|}
\hline $\begin{array}{l}\text { Nível de gravidade } \\
\text { - Micção }\end{array}$ & $\%$ & $\begin{array}{l}\text { Nível de gravidade } \\
\text { - Incontinência }\end{array}$ & $\%$ \\
\hline 1 & 6,3 & $\begin{array}{l}1 \\
\text { (0 episódio por dia) }\end{array}$ & 38,9 \\
\hline $\begin{array}{l}2 \\
\text { (8-10 episódios por dia) }\end{array}$ & 30,7 & $\begin{array}{l}2 \\
\text { (0-1 episódio por dia) }\end{array}$ & 18,8 \\
\hline $\begin{array}{l}3 \\
\text { (10-12 episódios por dia) }\end{array}$ & 27,2 & $\begin{array}{l}1 \\
\text { (1-2 episódios por dia) }\end{array}$ & 14,6 \\
\hline $\begin{array}{l}4 \\
\text { (12-14 episódios por dia) }\end{array}$ & 19,5 & $\begin{array}{l}1 \\
\text { (2-3 episódios por dia) }\end{array}$ & 9,2 \\
\hline $\begin{array}{l}5 \\
\text { (> } 14 \text { episódios por dia) }\end{array}$ & 16,4 & $\begin{array}{l}1 \\
\text { (3 episódios por dia) }\end{array}$ & 18,5 \\
\hline
\end{tabular}

\section{Probabilidades de transição}

As probabilidades de transição entre os níveis de gravidade da doença foram obtidos a partir de um modelo de regressão multinomial com dados do estudo SCORPIO e uma metanálise de comparação indireta que incluiu estudos clínicos com antimuscarínicos (fesoterodina, solifenacina, tolterodina, oxibutinina e tróspio) [Khullar et al., 2013; National Institute For Health and Care Excellence (NICE), 2013; Nazir et al., 2015]. Resultados do estudo SCORPIO indicam que a melhora dos sintomas da doença é mais evidente nas primeiras quatro semanas de tratamento, reduzindo progressivamente nas semanas seguintes. Dessa forma, adotou-se a premissa de que as probabilidades de transição iriam variar durante os primeiros três meses de tratamento e, após esse período, permaneceriam constantes ao longo dos nove meses seguintes (considerando um horizonte temporal de um ano).

\section{Descontinuação do tratamento}

Taxas de persistência específicas por tratamento foram extraídas de um estudo que avaliou esse parâmetro em pacientes com SBH no Reino Unido, conforme apresentado na Tabela 3 (Chapple et al., 2017).

Tabela 3. Persistência no tratamento (Chapple et al., 2017)

\begin{tabular}{lccc}
\hline Medicamento & Mês 1, \% & Mês 12, \% & $\begin{array}{c}\text { Longo } \\
\text { prazo, \% }\end{array}$ \\
\hline Mirabegrona & 77,1 & 38,5 & 31,2 \\
\hline Tolterodina & 63,8 & 24,3 & 18,5 \\
\hline Oxibutinina LI & 52,6 & 12,8 & 10,0 \\
\hline Oxibutinina LE & 59,8 & 17,6 & 13,4 \\
\hline Darifenacina (7,5/15 mg) & 63,8 & 16,0 & 12,4 \\
\hline Solifenacina (5/10 mg) & 65,1 & 25,6 & 19,0 \\
\hline
\end{tabular}

LI: liberação imediata; LE: liberação estendida.

\section{Comorbidades relacionadas à SBH}

O modelo considerou as comorbidades mais frequentes relacionadas à SBH: depressão, rash cutâneo e infecção do trato urinário - ITU (Arlandis-Guzman et al., 2011; Irwin et al., 2006). As probabilidades de rash e ITU em seis meses foram extraídas de Arlandis-Guzman et al. (2011) e convertidas em probabilidades mensais (Arlandis-Guzman et al., 2011). Já a probabilidade de depressão foi extraída de Irwin et al. (2006) apresentado na Tabela 4 (Irwin et al., 2006). A quantidade de absorventes diários para incontinência foi extraída do estudo SCORPIO (Khullar et al., 2013).

Tabela 4. Probabilidade mensal da ocorrência de comorbidades e uso diário de absorventes para incontinência (Irwin et al., 2006; Arlandis-Guzman et al., 2011; Khullar et al., 2013)

\begin{tabular}{lcccc}
\hline $\begin{array}{l}\text { Nível de } \\
\text { gravidade }\end{array}$ & Depressão & Rash & ITU & $\begin{array}{c}\text { Absorventes/ } \\
\text { dia }\end{array}$ \\
\hline Nível 1 & 0,011 & 0,016 & 0,035 & 0,170 \\
\hline Nível 2 & 0,012 & 0,017 & 0,040 & 0,750 \\
\hline Nível 3 & 0,015 & 0,018 & 0,050 & 1,380 \\
\hline Nível 4 & 0,017 & 0,019 & 0,059 & 1,890 \\
\hline Nível 5 & 0,021 & 0,020 & 0,074 & 3,340 \\
\hline
\end{tabular}

ITU: infecção do trato urinário.

\section{Carga anticolinérgica}

A carga anticolinérgica, definida como o efeito cumulativo de pelo menos um medicamento com ação anticolinérgica, está associada à ocorrência de diferentes eventos de acordo com o grau de exposição (Nery \& Reis, 2019). Uma análise do Regenstrief Medical Record System (RMRS), base de dados de informações médicas que abrange 1,3 milhão de pacientes do centro médico da Universidade de Indiana, demonstrou correlação positiva entre a utilização de antimuscarínicos e a carga anticolinérgica. Especificamente, um aumento de um ponto na carga anticolinérgica resultou em um incremento de 1,14 visita médica e 0,11 visita à emergência anualmente, ajustado por idade, gênero, raça e comorbidades (Campbell et al., 2016; Wielage et al., 2016). Segundo a escala brasileira de atividade anticolinérgica, os antimuscarínicos apresentam escore 3, enquanto o uso de mirabegrona não está associado a aumento da carga anticolinérgica (Nery \& Reis, 2019).

\section{Uso de recursos e custos}

Custos foram avaliados de acordo com a perspectiva do pagador (público e privado). O padrão de uso de recursos foi determinado por meio da opinião de especialistas e custeado de acordo com as tabelas apropriadas à perspectiva adotada [pública: Tabela SIGTAP (Sistema de Gerenciamento da Tabela de Procedimentos, Medicamentos); privada: CBHPM (Classificação Brasileira Hierarquizada de Procedimentos Médicos) 5 a edição com atualização de custos de acordo com os valores de porte 
de 2018]. O custo unitário dos absorventes para incontinência foi extraído do Banco de Preços em Saúde, para a perspectiva pública, e do preço médio do item em farmácias, conforme busca conduzida na internet. Custos de medicamentos foram extraídos da lista da Câmara de Regulação do Mercado de Medicamentos, considerando o preço-fábrica (PF) ou o preço máximo de venda ao governo (PMVG), com 18\% de ICMS, para as perspectivas privada e pública, respectivamente. Os custos consideraram a média de preços, incluindo medicamentos de referência e genéricos disponíveis no mercado.

A Tabela 5 apresenta os custos de aquisição de medicamentos utilizados na análise. Já a Tabela 6 apresenta os custos com manejo de comorbidades e o custo unitário de consultas, visita à emergência e absorventes para incontinência. Os parâmetros utilizados para o cálculo de custos estão apresentados como material suplementar.

Tabela 5. Custo mensal com aquisição de medicamentos

\begin{tabular}{lcc}
\hline Medicamento & $\begin{array}{c}\text { Privada, R\$ } \\
\text { (PF 18\%) }\end{array}$ & $\begin{array}{c}\text { Pública, R\$ } \\
\text { (PMVG 18\%) }\end{array}$ \\
\hline Mirabegrona & 182,20 & 145,47 \\
\hline Tolterodina & 320,46 & 255,86 \\
\hline Oxibutinina LI & 37,91 & 30,27 \\
\hline Oxibutinina LE & 75,32 & 60,14 \\
\hline Darifenacina (7,5/15 mg) & 190,90 & 152,34 \\
\hline Solifenacina (5 mg) & 115,01 & 91,83 \\
\hline Solifenacina (10 mg) & 140,73 & 112,36 \\
\hline $\begin{array}{l}\text { LI: liberação imediata; LE: liberação estendida; PF: preço-fábrica; PMVG: preço má- } \\
\text { ximo de venda ao governo. }\end{array}$ & &
\end{tabular}

Tabela 6. Custo de comorbidades

\begin{tabular}{lcc}
\hline Item de custo & Privada, R\$ & Pública, R\$ \\
\hline Manejo de depressão & 364,09 & 11,57 \\
\hline Manejo de rash & 115,89 & 15,35 \\
\hline $\begin{array}{l}\text { Manejo da infecção } \\
\text { do trato urinário }\end{array}$ & 364,98 & 41,95 \\
\hline Consulta (custo unitário) & 75,32 & 10,00 \\
\hline Visita à emergência (diária) & 460,06 & 12,47 \\
\hline Absorventes para incontinência & 2,90 & 1,30 \\
\hline
\end{tabular}

\section{Análise de sensibilidade}

O impacto das incertezas nos resultados do caso-base da análise foi avaliado por meio de análise de sensibilidade determinística.

\section{Resultados}

\section{Perspectiva do sistema de saúde suplementar}

Os resultados de custo-efetividade para a perspectiva do sistema de saúde suplementar no horizonte de tempo de um ano, considerando uma taxa de desconto de 5\% para custos e desfechos, estão apresentados na Tabela 7. A Tabela 8 apresenta os resultados incrementais, enquanto a Tabela 9 apresenta a RCEl. Em termos gerais, o tratamento com mirabegrona apre-

Tabela 7. Resultados da análise de custo-efetividade (horizonte temporal de 1 ano), sob a perspectiva do sistema de saúde suplementar

\begin{tabular}{lccc}
\hline Medicamento & $\begin{array}{c}\text { Custo } \\
\text { total, R\$ }\end{array}$ & $\begin{array}{c}\text { Melhora } \\
\text { do nível de } \\
\text { incontinência }\end{array}$ & $\begin{array}{c}\text { Melhora } \\
\text { do nível de } \\
\text { micção }\end{array}$ \\
\hline Mirabegrona & $2.455,26$ & 0,491 & 0,498 \\
\hline Tolterodina & $3.196,27$ & 0,303 & 0,291 \\
\hline Oxibutinina LI & $1.707,24$ & 0,168 & 0,160 \\
\hline Oxibutinina LE & $1.881,51$ & 0,229 & 0,210 \\
\hline Darifenacina & $2.402,36$ & 0,210 & 0,201 \\
\hline $\begin{array}{l}\text { Solifenacina } \\
\text { (5 mg) }\end{array}$ & $2.134,98$ & 0,326 & 0,187 \\
\hline $\begin{array}{l}\text { Solifenacina } \\
\text { (10 mg) }\end{array}$ & $2.274,08$ & 0,326 & 0,311 \\
\hline LI: liberação imediata; LE: liberação estendida. & & \\
\hline
\end{tabular}

Tabela 8. Resultados incrementais, sob a perspectiva do sistema de saúde suplementar

\begin{tabular}{lccc}
\hline Medicamento & $\begin{array}{c}\text { Custo } \\
\text { incremental, } \\
\mathbf{R} \$\end{array}$ & $\begin{array}{c}\text { Melhora } \\
\text { do nível de } \\
\text { incontinência }\end{array}$ & $\begin{array}{c}\text { Melhora } \\
\text { do nível de } \\
\text { micção }\end{array}$ \\
\hline Mirabegrona & - & - & - \\
\hline Tolterodina & $-741,01$ & 0,188 & 0,207 \\
\hline Oxibutinina LI & 748,02 & 0,168 & 0,338 \\
\hline Oxibutinina LE & 573,75 & 0,262 & 0,280 \\
\hline Darifenacina & 52,90 & 0,210 & 0,298 \\
\hline $\begin{array}{l}\text { Solifenacina } \\
\text { (5 mg) }\end{array}$ & 320,28 & 0,164 & 0,187 \\
\hline $\begin{array}{l}\text { Solifenacina } \\
\text { (10 mg) }\end{array}$ & 181,17 & 0,164 & 0,187 \\
\hline
\end{tabular}

LI: liberação imediata; LE: liberação estendida.

Tabela 9. Razão de custo-efetividade incremental, sob a perspectiva do sistema de saúde suplementar

\begin{tabular}{lcc}
\hline Medicamento & $\begin{array}{c}\text { RCEI (R\$/melhora } \\
\text { do nível de } \\
\text { incontinência) }\end{array}$ & $\begin{array}{c}\text { RCEI (R\$/melhora } \\
\text { do nível de micção) }\end{array}$ \\
\hline Mirabegrona & - & - \\
\hline Tolterodina & Dominante & Dominante \\
\hline Oxibutinina LI & $2.317,14$ & $2.211,57$ \\
\hline Oxibutinina LE & $2.189,57$ & $2.046,91$ \\
\hline Darifenacina & 188,73 & 177,69 \\
\hline Solifenacina (5 mg) & $1.949,69$ & $1.711,59$ \\
\hline Solifenacina (10 mg) & $1.102,88$ & 968,19 \\
\hline LI: liberação imediata; LE: liberação estendida; RCEl: razão de custo-efetividade \\
incremental
\end{tabular}
incremental. 
sentou maior efetividade quanto à melhora dos níveis de gravidade da incontinência e frequência de micção. Em relação aos custos, o tratamento foi dominante quando comparado à tolterodina e custo-efetivo versus os demais comparadores.

\section{Perspectiva do sistema de saúde público}

Assim como para o sistema de saúde suplementar, a mirabegrona apresentou maior efetividade quanto à melhora dos níveis de gravidade da incontinência e frequência de micção, sendo dominante em relação à tolterodina e custo-efetiva quanto aos demais comparadores. A Tabela 10 apresenta os resultados de custo-efetividade, enquanto a Tabela 11 e a Tabela 12 apresentam os resultados incrementais e a RCEl, respectivamente.

\section{Análise de sensibilidade determinística}

Os parâmetros de maior influência em relação aos resultados, independentemente da perspectiva, foram as taxas de persistência em 1 e 12 meses para mirabegrona e seus comparadores. No entanto, em nenhuma das comparações ou perspectivas a variação desse parâmetro foi capaz de alterar de maneira significativa os resultados da análise.

\section{Discussão}

Este estudo foi conduzido com o objetivo de determinar a relação de custo-efetividade de mirabegrona como tratamento de primeira escolha para SBH, utilizando como comparadores os antimuscarínicos orais comumente prescritos no manejo de pacientes adultos no Brasil. Os antimuscarínicos disponíveis e incluídos na avaliação foram tolterodina, oxibutinina, darifenacina e solifenacina. Foram considerados como pagadores o sistema de saúde público e o privado. A condução de análises econômicas para a avaliação de tecnologias em saúde é capaz de auxiliar no processo de tomada de decisão, uma vez que pondera a relação entre custos decorrentes de sua adoção e os benefícios clínicos (Secoli et al., 2010).

O sistema brasileiro de saúde é atualmente dividido em duas estratégias principais, o sistema público, por meio do Sistema Único de Saúde (SUS), e o sistema privado, por meio do sistema de saúde suplementar (Paim et al., 2011; Pietrobon et al., 2008). O sistema brasileiro de saúde suplementar atende aproximadamente 47 milhões de brasileiros, correspondendo a cerca de $24 \%$ da população. O restante da população recebe assistência por meio do SUS [Agência Nacional de Saúde Suplementar (ANS), 2019]. Dessa forma, este estudo traz uma visão global do tratamento farmacológico da SBH, sob a perspectiva da assistência à saúde no Brasil, uma vez que ambos os tipos de financiamento são analisados.

Em estudos de farmacoeconomia, realiza-se uma análise em que os custos de determinada tecnologia são confron-
Tabela 10. Resultados da análise de custo-efetividade (horizonte temporal de 1 ano), sob a perspectiva do pagador público

\begin{tabular}{lccc}
\hline Medicamento & $\begin{array}{c}\text { Custo } \\
\text { total, R\$ }\end{array}$ & $\begin{array}{c}\text { Melhora } \\
\text { do nível de } \\
\text { incontinência }\end{array}$ & $\begin{array}{c}\text { Melhora } \\
\text { do nível de } \\
\text { micção }\end{array}$ \\
\hline Mirabegrona & $1.396,01$ & 0,491 & 0,498 \\
\hline Tolterodina & $1.814,90$ & 0,303 & 0,291 \\
\hline Oxibutinina LI & 626,24 & 0,168 & 0,160 \\
\hline Oxibutinina LE & 765,21 & 0,229 & 0,210 \\
\hline Darifenacina & $1.180,95$ & 0,210 & 0,201 \\
\hline $\begin{array}{l}\text { Solifenacina } \\
\text { (5 mg) }\end{array}$ & 967,38 & 0,326 & 0,187 \\
\hline $\begin{array}{l}\text { Solifenacina } \\
\text { (10 mg) }\end{array}$ & $1.078,41$ & 0,326 & 0,311 \\
\hline
\end{tabular}

LI: liberação imediata; LE: liberação estendida.

Tabela 11. Resultados incrementais, sob a perspectiva do pagador público

\begin{tabular}{lccc}
\hline Medicamento & $\begin{array}{c}\text { Custo } \\
\text { incremental, } \\
\mathbf{R} \$\end{array}$ & $\begin{array}{c}\text { Melhora } \\
\text { do nível de } \\
\text { incontinência }\end{array}$ & $\begin{array}{c}\text { Melhora } \\
\text { do nível de } \\
\text { micção }\end{array}$ \\
\hline Mirabegrona & - & - & - \\
\hline Tolterodina & $-418,89$ & 0,188 & 0,207 \\
\hline Oxibutinina LI & 769,77 & 0,168 & 0,338 \\
\hline Oxibutinina LE & 630,80 & 0,262 & 0,280 \\
\hline Darifenacina & 215,06 & 0,210 & 0,298 \\
\hline $\begin{array}{l}\text { Solifenacina } \\
\text { (5 mg) }\end{array}$ & 428,63 & 0,164 & 0,187 \\
\hline $\begin{array}{l}\text { Solifenacina } \\
\text { (10 mg) }\end{array}$ & 317,60 & 0,164 & 0,187 \\
\hline
\end{tabular}

LI: liberação imediata; LE: liberação estendida.

Tabela 12. Razão de custo-efetividade incremental, sob a perspectiva do pagador público

\begin{tabular}{lcc}
\hline Medicamento & $\begin{array}{c}\text { RCEI (R\$/melhora } \\
\text { do nível de } \\
\text { incontinência) }\end{array}$ & $\begin{array}{c}\text { RCEI (R\$/melhora } \\
\text { do nível de micção) }\end{array}$ \\
\hline Mirabegrona & - & - \\
\hline Tolterodina & Dominante & Dominante \\
\hline Oxibutinina LI & $2.384,54$ & $2.275,89$ \\
\hline Oxibutinina LE & $2.407,31$ & $2.250,46$ \\
\hline Darifenacina & 767,29 & 722,43 \\
\hline Solifenacina (5 mg) & $2.609,30$ & $2.290,64$ \\
\hline Solifenacina (10 mg) & $1.933,36$ & $1.697,25$ \\
\hline
\end{tabular}

LI: liberação imediata; LE: liberação estendida; RCEl: razão de custo-efetividade incremental.

tados com seus benefícios clínicos, obtendo-se a relação de custo-efetividade. Uma tecnologia pode ser considerada custo-efetiva quando é observado um melhor desfecho clínico, porém com maior custo, desde que o custo incremental es- 
teja abaixo do limiar de disposição a pagar. A intervenção é dita dominante em relação ao seu comparador quando gera economia de recursos, além de ganhos em termos de eficácia. Ainda, pode ser considerada dominada quando gera custo incremental e pior desfecho clínico (Secoli et al., 2010).

Na presente análise, a mirabegrona mostrou-se dominante em relação ao uso de tolterodina, sob as perspectivas pública e privada. Estudos conduzidos na Colômbia, Japão, Estados Unidos e Reino Unido demonstraram o custo-efetividade da mirabegrona quando comparada à tolterodina (Aballéa et al., 2014; Nazir et al., 2015; Parise et al., 2020; Wielage et al., 2016; Yamanishi et al., 2018). Em uma análise conduzida nos Estados Unidos, a mirabegrona se mostrou dominante em relação à tolterodina (Wielage et al., 2016).

Quando considerados os outros comparadores incluídos nesta análise (oxibutinina, darifenacina e solifenacina), a mirabegrona não se mostrou dominante (menor custo e maior efetividade), porém foi custo-efetiva (maior efetividade e custo incremental abaixo do limiar de disposição a pagar) quando considerado um limite de disposição a pagar de um PIB per capita (R\$32.747), corroborando achados previamente descritos (Nazir et al., 2015; Parise et al., 2020; Wielage et al., 2016). Wielage et al. (2016) observaram a dominância da mirabegrona quando comparada à solifenacina, avaliada sob a perspectiva do sistema americano de saúde. Uma possível explicação para essa diferença está no horizonte temporal adotado. No presente estudo, um período de apenas um ano foi adotado, enquanto Wielage et al. (2016) utilizam um horizonte temporal de três anos (Wielage et al., 2016).

Maiores taxas de persistência do tratamento com mirabegrona comparada aos antimuscarínicos foram relatadas (Chapple et al., 2017; Kato et al., 2017; Wagg et al., 2015). Essa diferença é o fator com maior influência nos resultados desta análise. A persistência no tratamento determina a distribuição dos pacientes por nível de gravidade da doença, no decorrer do horizonte de um ano. Adicionalmente, o nível de gravidade de incontinência está associado à ocorrência de comorbidades comuns à SBH, as quais foram incluídas no modelo: depressão, rash cutâneo e ITU. Para ajustar as probabilidades das comorbidades à gravidade da incontinência, elas foram transformadas de uma distribuição binária (controlada/não controlada) para uma distribuição contínua, assumindo uma relação linear com os episódios de incontinência e assumindo que o maior nível de gravidade nesse modelo foi o estado não controlado, seguindo o mesmo racional descrito por estudo conduzido na Colômbia (Parise et al., 2020). Outro fator importante no modelo foi a estimativa do impacto da carga anticolinérgica, associada ao uso de antimuscarínicos, mas não ao de mirabegrona (Nery \& Reis, 2019). O escore de carga anticolinérgica foi diretamente associado com a utilização de recursos em saúde e risco de deterioração cognitiva (Campbell et al., 2016).
Os resultados desta análise sugerem que a mirabegrona é custo-efetiva comparada aos antimuscarínicos, sob a perspectiva dos sistemas de saúde suplementar e público. O maior custo com a aquisição de mirabegrona em relação aos comparadores foi compensado por sua maior efetividade quanto à melhora dos níveis de gravidade da incontinência e frequência de micção, maior persistência, menos comorbidades relacionadas à SBH, ausência de carga anticolinérgica e menor utilização de absorventes para incontinência. Custos com o manejo de EAs comuns a essas medicações, como boca seca e constipação, não foram incluídos na análise, já que eles podem ser considerados na análise dos custos com o aumento da carga anticolinérgica. Sob a perspectiva dos pacientes, a ocorrência desses EAs gera significativo incômodo e é uma razão comum para a descontinuação do tratamento (Kelleher et al., 2018). A melhora de desfechos clínicos tem influência na adesão ao tratamento e observou-se redução de custos com pacientes que persistem com o tratamento para SBH comparados àqueles que trocam de medicação antimuscarínica (Ivanova et al., 2014).

Custos indiretos com impacto populacional também não foram incluídos, como a perda de produtividade. A SBH está associada à maior ocorrência de absenteísmo e redução da produtividade no trabalho. No estudo Brasil LUTS, $2 \%$ dos homens e 6,5\% das mulheres relataram faltar ao trabalho devido à $\mathrm{SBH}$. O impacto da doença no âmbito pessoal é ainda mais significativo, $14,8 \%$ dos homens e $25,8 \%$ das mulheres reportaram que a SBH leva à perda de atividades de lazer e/ou tem impacto negativo nas atividades diárias (Gomes et al., 2020). Portanto, há um nível significativo de custos indiretos e intangíveis para o paciente com SBH, que podem ser amenizados com melhor persistência do tratamento.

Apesar dos achados relevantes apresentados, este estudo apresenta limitações. Os dados utilizados na construção do modelo são oriundos de estudos internacionais, uma vez que não há estudos conduzidos na população brasileira para alguns dos desfechos considerados, podendo influenciar as estimativas geradas. Além disso, o modelo assume que pacientes que descontinuam o tratamento para SBH permanecem sem tratamento durante o horizonte de um ano, ou seja, sem iniciar novo tratamento farmacológico ou mesmo sem retornar ao tratamento inicial. Embora, dessa forma, o impacto causado por diferentes sequências de tratamento em custos e desfechos possa ser minimizado, padrões de tratamento para SBH no Brasil podem não estar acuradamente refletidos no modelo. Além disso, determinou-se o custo-efetividade da intervenção com base em um limiar de disposição a pagar equivalente a um PIB per capita. Porém, sabe-se que a determinação de custo-efetividade de uma intervenção é decorrente de múltiplos fatores e não deve ser determinada apenas por um limiar fixo predeterminado. 


\section{Conclusão}

A análise de custo-efetividade do uso de mirabegrona como primeira escolha de tratamento farmacológico para a SBH demonstrou dominância desse agente quando comparado à tolterodina, um agente antimuscarínico oral, ou seja, apresentando maior efetividade e menor custo, tanto na perspectiva do sistema de saúde público como na do sistema de saúde suplementar. Além disso, a mirabegrona demonstrou ser custo-efetiva na comparação com os demais agentes antimuscarínicos: oxibutinina, darifenacina e solifenacina. Dessa forma, é possível concluir que a mirabegrona pode ser considerada a melhor opção para o tratamento farmacológico da SBH, com potencial de redução de custos ao longo do tempo, sob as perspectivas do sistema de saúde suplementar e público no Brasil.

\section{Agradecimentos}

Agradecemos à SENSE Company pelo apoio com o desenvolvimento do modelo econômico e a redação científica durante o desenvolvimento deste manuscrito. Esse apoio foi financiado pela Astellas.

\section{MATERIAL SUPLEMENTAR}

Parâmetros considerados para o cálculo do custo da depressão, perspectiva pública

\begin{tabular}{|c|c|c|c|c|c|c|}
\hline & $\%$ em uso & Quantidade & Custo unitário & Custo total & Fonte & Código \\
\hline \multicolumn{7}{|l|}{ Custo médico } \\
\hline Consulta com especialista & $100 \%$ & 1 & $R \$ 10,00$ & $R \$ 10,00$ & SIGTAP 02/2019 & 03.01.01.007-2 \\
\hline \multicolumn{7}{|l|}{ Custo farmacêutico } \\
\hline Fluoxetina & $100 \%$ & 30 & $R \$ 0,05$ & $R \$ 1,57$ & BPS, 06/2019 & BR0273009 \\
\hline TOTAL & & & & $\mathrm{R} \$ 11,57$ & & \\
\hline
\end{tabular}

Parâmetros considerados para o cálculo do custo da depressão, perspectiva do sistema de saúde suplementar

\begin{tabular}{lcccccc}
\hline & \% em uso & Quantidade & Custo unitário & Custo total & Fonte & Código \\
\hline Custo médico & & & & & & \\
\hline Consulta com especialista & $100 \%$ & 3 & $R \$ 104,64$ & $R \$ 313,92$ & CBHPM 2018 & $1.01 .01 .01-2$ \\
\hline Fluoxetina $-20 \mathrm{mg} /$ dia & $100 \%$ & 30 & $R \$ 1,67$ & $R \$ 50,17$ & $\begin{array}{c}\text { CMED 09/19, } \\
\text { PF 18\% }\end{array}$ & \begin{tabular}{c}
507602307111319 \\
\hline
\end{tabular} \\
\hline
\end{tabular}

TOTAL

$\mathrm{R} \$ 364,09$

Parâmetros considerados para o cálculo do custo do rash, perspectiva pública

\begin{tabular}{|c|c|c|c|c|c|c|}
\hline & $\%$ em uso & Quantidade & Custo unitário & Custo total & Fonte & Código \\
\hline Consulta com especialista & $100 \%$ & 1 & $\mathrm{R} \$ 10,00$ & $\mathrm{R} \$ 10,00$ & SIGTAP 02/2019 & 03.01.01.007-2 \\
\hline Subtotal do custo médico & & & & $R \$ 10,00$ & & \\
\hline \multicolumn{7}{|l|}{ Custo farmacêutico } \\
\hline Hidrocortisona creme & $50 \%$ & 2 & $R \$ 5,35$ & $R \$ 5,35$ & BPS, 06/2019 & BR0268851 \\
\hline CUSTO TOTAL & & & & $\mathrm{R} \$ 15,35$ & & \\
\hline
\end{tabular}

Parâmetros considerados para o cálculo do custo do rash, perspectiva do sistema de saúde suplementar

\begin{tabular}{|c|c|c|c|c|c|c|}
\hline & $\%$ em uso & Quantidade & Custo unitário & Custo total & Fonte & Código \\
\hline Consulta com especialista & $100 \%$ & 1 & $\mathrm{R} \$ 104,64$ & $\mathrm{R} \$ 104,64$ & CBHPM 2018 & $1.01 .01 .01-2$ \\
\hline Hidrocortisona creme & $50 \%$ & 1 & $\mathrm{R} \$ 22,49$ & $\mathrm{R} \$ 11,25$ & $\begin{array}{c}\text { CMED 09/19, } \\
\text { PF 18\% }\end{array}$ & 538912050013813 \\
\hline
\end{tabular}


Parâmetros considerados para o cálculo do custo da infecção do trato urinário, perspectiva pública

\begin{tabular}{|c|c|c|c|c|c|c|}
\hline & Frequência & Quantidade & Custo unitário & Custo total & Fonte & Código \\
\hline \multicolumn{7}{|l|}{ Custo médico } \\
\hline Consulta médica & $100 \%$ & 2 & $\mathrm{R} \$ 10,00$ & $R \$ 20,00$ & SIGTAP 02/2019 & 03.01.01.007-2 \\
\hline Urinocultura & $100 \%$ & 2 & $R \$ 5,62$ & $\mathrm{R} \$ 11,24$ & SIGTAP 02/2019 & 02.02.08.008-0 \\
\hline Subtotal do custo médico & & & & $R \$ 38,64$ & & \\
\hline $\begin{array}{l}\text { Norfloxacino } 400 \text { mg duas } \\
\text { vezes ao dia/7 dias }\end{array}$ & $100 \%$ & 14 & $R \$ 0,24$ & $R \$ 3,31$ & BPS, 10/2019 & BR0305270 \\
\hline Subtotal do custo farmacêutico & & & & $R \$ 3,31$ & & \\
\hline TOTAL & & & & $\mathrm{R} \$ 41,95$ & & \\
\hline
\end{tabular}

Parâmetros considerados para o cálculo do custo da infecção do trato urinário, perspectiva do sistema de saúde suplementar

\begin{tabular}{|c|c|c|c|c|c|c|}
\hline & Frequência & Quantidade & Custo unitário & Custo total & Fonte & Código \\
\hline \multicolumn{7}{|l|}{ Custo médico } \\
\hline Consulta médica & $100 \%$ & 2 & $R \$ 104,64$ & $R \$ 209,28$ & CBHPM 2018 & 1.01.01.01-2 \\
\hline Urinocultura & $100 \%$ & 2 & $R \$ 37,64$ & $R \$ 75,28$ & CBHPM 2018 & 4.03.10.21-3 \\
\hline $\begin{array}{l}\text { Ciprofloxacino - } 500 \\
\text { mg/dia por } 7 \text { dias }\end{array}$ & $100 \%$ & 7 & $R \$ 6,53$ & $R \$ 45,68$ & $\begin{array}{c}\text { CMED 09/19, } \\
\text { PF 18\% }\end{array}$ & 538915100024417 \\
\hline
\end{tabular}

TOTAL

$\mathrm{R} \$ 364,98$

Parâmetros considerados para o cálculo do custo de visitas médicas e pads para incontinência, perspectiva pública

\begin{tabular}{lccc}
\hline & Custo unitário & Fonte & Código \\
\hline Consulta em serviço de atenção primária & R\$ 10,00 & SIGTAP 02/2019 & $03.01 .01 .007-2$ \\
\hline Consulta ambulatorial & R\$ 10,00 & SIGTAP 02/2019 & $03.01 .01 .007-2$ \\
\hline Atendimento em serviço de emergência & R\$ 12,47 & SIGTAP 02/2019 & $03.01 .06 .002-9$ \\
\hline Absorventes para incontinência & R\$ 1,30 & BPS, 06/2019 & BR0358131 \\
\hline
\end{tabular}

Parâmetros considerados para o cálculo do custo de visitas médicas e pads para incontinência, perspectiva do sistema de saúde suplementar

\begin{tabular}{lccc}
\hline & Custo unitário & Fonte & Código \\
\hline Consulta em serviço de atenção primária & $\mathrm{R} \$ 104,64$ & $\mathrm{CBHPM} 2018$ & $1.01 .01 .01-2$ \\
\hline Consulta ambulatorial & $\mathrm{R} \$ 104,64$ & $\mathrm{CBHPM} 2018$ & $1.01 .01 .01-2$ \\
\hline Atendimento em serviço de emergência & $\mathrm{R} \$ 460,06$ & Planserv & $71.00 .118-1$ \\
\hline Absorventes para incontinência & $\mathrm{R} \$ 2,90$ & & \\
\hline
\end{tabular}

\section{Referências bibliográficas}

Aballéa S, Maman K, Thokagevistk K, Nazir J, Odeyemi IA, Hakimi Z, et al. Cost effectiveness of mirabegron compared with tolterodine extended release for the treatment of adults with overactive bladder in the United Kingdom. Clin Drug Investig. 2014;35(2):83-93.

Abrams P, Kelleher CJ, Kerr LA, Rogers RG. Overactive bladder significantly affects quality of life. Am J Manag Care. 2000;6(11 Suppl):S580-90.

Agência Nacional de Saúde Suplementar (ANS). Sala de Situação. 2019. Available from: http://www.ans.gov.br/perfil-do-setor/dados-eindicadores-do-setor/sala-de-situacao. Accessed on: Set 30, 2019.
Arlandis-Guzman S, Errando-Smet C, Trocio J, Arumi D, Rejas J. Costeffectiveness analysis of antimuscarinics in the treatment of patients with overactive bladder in Spain: A decision-tree model. BMC Urol. 2011;1:9.

Campbell NL, Perkins AJ, Bradt P, Perk S, Wielage RC, Boustani MA, et al. Association of Anticholinergic Burden with Cognitive Impairment and Health Care Utilization Among a Diverse Ambulatory Older Adult Population. Pharmacotherapy. 2016;36(11):1123-31.

Chapple CR, Nazir J, Hakimi Z, Bowditch S, Fatoye F, Guelfucci F, et al. Persistence and Adherence with Mirabegron versus Antimuscarinic Agents in Patients with Overactive Bladder: A Retrospective Observational Study in UK Clinical Practice. Eur Urol. 2017;72(3):389-99. 
Corcos J, Przydacz M, Campeau L, Witten J, Hickling D, Honeine C, et al. CUA guideline on adult overactive bladder. Can Urol Assoc J. 2017;11(5):E142-73.

Deeks ED. Mirabegron: A Review in Overactive Bladder Syndrome. Drugs. 2018;78(8):833-44.

Gomes CM, Averbeck MA, Koyama M, Soler R. Association Among Lower Urinary Tract Symptoms, Erectile Function, and Sexual Satisfaction: Results from the Brazil LUTS Study. Sex Med. 2020;8(1):45-56.

Gomes CM, Averbeck MA, Koyama M, Soler R. Impact of OAB symptoms on work, quality of life and treatment-seeking behavior in Brazil [published online ahead of print, 2020 Apr 24]. Curr Med Res Opin. 2020;1.

International Continence Society (ICS). Overactive Bladder. 2018. Available from: https://www.ics.org/committees/standardisation/ terminologydiscussions/overactivebladder\#targetText=In. Accessed on: Dec 10, 2019.

Irwin D, Milsom I, Reilly K, Hunskaar S, Coyne KS, Kopp Z, et al. Men and women with overactive bladder symptoms report higher prevalence of depression and lower quality of life: Results from the EPIC study. Neurourol Urodynamics. 2006;25(6).

Irwin DE, Mungapen L, Milsom I, Kopp Z, Reeves P, Kelleher C. The economic impact of overactive bladder syndrome in six Western countries. BJU Int. 2009;103(2):202-9.

Irwin DE, Kopp ZS, Agatep B, Milsom I, Abrams P. Worldwide prevalence estimates of lower urinary tract symptoms, overactive bladder, urinary incontinence and bladder outlet obstruction. BJU Int. 2011;108(7):1132-9.

Ivanova Jl, Hayes-Larson E, Sorg RA, Birnbaum HG, Berner T. Healthcare resource use and costs of privately insured patients who switch, discontinue, or persist on anti-muscarinic therapy for overactive bladder. J Med Econ. 2014;17(10):741-50.

Johnston KM, Walker DR, Lakzadeh P. Characterizing the Health-Related Quality of Life Burden of Overactive Bladder Using Disease-Specific Patient-Reported Outcome Measures: A Systematic Literature Review. Adv Ther. 2019;36(3):548-62

Kato D, Uno S, Van Schyndle J, Fan A, Kimura T. Persistence and adherence to overactive bladder medications in Japan: A large nationwide real-world analysis. Int J Urol. 2017;24(10):757-64.

Kelleher C, Hakimi Z, Zur R, Siddiqui E, Maman K, Aballéa S, et al. Efficacy and Tolerability of Mirabegron Compared with Antimuscarinic Monotherapy or Combination Therapies for Overactive Bladder: A Systematic Review and Network Meta-analysis. Eur Urol. 2018;74(3):324-33.

Khullar V, Amarenco G, Angulo JC, Cambronero J, Høye K, Milsom I, et al. Efficacy and tolerability of mirabegron, a $\beta(3)$-adrenoceptor agonist, in patients with overactive bladder: results from a randomised EuropeanAustralian phase 3 trial. Eur Urol. 2013;63(2):283-95.

Leron E, Weintraub AY, Mastrolia SA, Schwarzman P. Overactive Bladder Syndrome: Evaluation and Management. Curr Urol. 2018;11(3):117-25.

Lightner DJ, Gomelsky A, Souter L, Vasavada SP. Diagnosis and Treatment of Overactive Bladder (Non-Neurogenic) in Adults: AUA/SUFU Guideline Amendment 2019. J Urol. 2019;202(3):558-63.

National Institute For Health and Care Excellence (NICE). Mirabegron for treating symptoms of overactive bladder. 2013. p. 1-50. Available from: guidance.nice.org.uk/ta290.
Nazir J, Maman K, Neine ME, Briquet B, Odeyemi IA, Hakimi Z, et al. CostEffectiveness of Mirabegron Compared with Antimuscarinic Agents for the Treatment of Adults with Overactive Bladder in the United Kingdom. Value Health. 2015;18(6):783-90.

Nery RT, Reis AMM. Development of a Brazilian anticholinergic activity drug scale. Einstein (São Paulo). 2019;17(2):1-6.

Nitti VW, Khullar V, van Kerrebroeck P, et al. Mirabegron for the treatment of overactive bladder: a prespecified pooled efficacy analysis and pooled safety analysis of three randomised, double-blind, placebo-controlled, phase III studies. Int J Clin Pract. 2013;67(7):619-32.

Paim J, Travassos C, Almeida C, Bahia L, Macinko J. The Brazilian health system: History, advances, and challenges. Lancet. 2011;377(9779):1778-97.

Parise H, Espinosa R, Dea K, Anaya P, Montoya G, Ng DB. Cost Effectiveness of Mirabegron Compared with Antimuscarinic Agents for the Treatment of Adults with Overactive Bladder in Colombia. Pharmacoeconom Open. 2020;4(1):79-90.

Pietrobon L, Prado ML, Caetano JC. Saúde suplementar no Brasil: o papel da Agência Nacional de Saúde Suplementar na regulação do setor. Physis. 2008;18(4):767-83.

Powell LC, Szabo SM, Walker D, Gooch K. The economic burden of overactive bladder in the United States: A systematic literature review. Neurourol Urodyn. 2018;37(4):1241-9.

Ricetto Sacomani CA, De Almeida FG, Silvinato A, Bernardo wm. Overactive bladder - pharmacological treatment. Rev Assoc Med Bras. 2019;65(4):487-92.

Secoli SR, Nita ME, Ono-Nita SK, Nobre M. Avaliação de tecnologia em saúde II. A análise de custo-efetividade. Arq Gastroenterol. 2010;47(4).

Soler R, Averbeck MA, Koyama MAH, Gomes CM. Impact of LUTS on treatmentrelated behaviors and quality of life: A population-based study in Brazil. Neurourol Urodyn. 2019;38(6):1579-87.

Soler R, Gomes CM, Averbeck MA, Koyama M. The prevalence of lower urinary tract symptoms (LUTS) in Brazil: Results from the epidemiology of LUTS (Brazil LUTS) study. Neurourol Urodyn. 2018;37(4):1356-64.

Tubaro A. Defining overactive bladder: Epidemiology and burden of disease. Urology. 2004;64(6 Suppl 1):2-6.

Wagg AS, Foley S, Peters J, Nazir J, Kool-Houweling L, Scrine L. Persistence and adherence with mirabegron vs antimuscarinics in overactive bladder: Retrospective analysis of a UK General Practice prescription database. Int J Clin Pract. 2017;71(10).

Wagg A, Franks B, Ramos B, Berner T. Persistence and adherence with the new beta-3 receptor agonist, mirabegron, versus antimuscarinics in overactive bladder: Early experience in Canada. J Can Urol Assoc. 2015;9(9-10):343-50.

Wielage RC, Perk S, Campbell NL, Klein TM, Posta LM, Yuran T, et al. Mirabegron for the treatment of overactive bladder: cost-effectiveness from US commercial health-plan and Medicare Advantage perspectives. J Med Econ. 2016;19(12):1135-43.

Yamanishi Y, Yamanishi T, Tajima H, Ikeda S. Mirabegron or tolterodine for the treatment of overactive bladder in Japan: Which drug is more costeffective as the first-line treatment? Int J Urol. 2018;25(10):863-70. 